\title{
INTUBAÇÃO SUBMENTONIANA: REVISÃO DE LITERATURA E DESCRIÇÃO DA TÉCNICA CIRÚRGICA
}

SUBMENTAL INTUBATION: LITERATURE REVIEW AND DESCRIPTION OF THE

SURGICAL TECHNIQUE

\section{Walace Daflon de Faria}

Aluno do $8^{\circ}$ período da Faculdade de Odontologia da Universidade Federal Fluminense / RJ.

\section{Adriano Campos}

Aluno do curso de especialização em Implantodontia da Universidade Veiga de Almeida /RJ

Mestrando em Prótese dentária pela Universidade do Estado do Rio de Janeiro / RJ.

\section{Eugênio Braz Rodrigues}

Especialista em Estomatologia pela Universidade Federal do Rio de Janeiro /RJ. Residente em Cirurgia e Traumatologia Buco-Maxilo-Facial do Hospital Federal dos Servidores do Estado e Universidade Federal Fluminense / RJ.

\section{Marcelo José Uzeda}

Mestre e Doutor em Odontologia pela Universidade Federal Fluminense / RJ. Professor da disciplina de Cirurgia Bucal da Universidade Iguaçu / RJ.

\section{Rodrigo Figueiredo de Brito Resende}

Mestre e Doutor em Odontologia pela Universidade Federal Fluminense / RJ. Professor da disciplina de Cirurgia Bucal da Universidade Iguaçu / RJ.

Universidade Federal Fluminense

Faculdade de Odontologia

Artigo de Revisão de literatura

Walace Daflon De Faria

Endereço: Rua Mario Santos Braga, 28 - Centro, Niterói - RJ, 24020-140

Telefone: (21) 2629-9920

Email: walace.faria@gmail.com 


\section{RESUMO}

A intubação submentoniana ou também conhecida como submento-oro-traqueal é uma técnica que conquistou diversos cirurgiões maxilofaciais ao longo dos anos, que por sua vez realizaram algumas pequenas alterações de acordo com seus conhecimentos anatômicos e vivência profissional. É um procedimento considerado seguro, fácil, rápido e de baixa morbidade. Por esse motivo é utilizada como alternativa para a traqueostomia, largamente utilizada e que possui maiores chances de complicações pósoperatórias. A intubação esta indicada para cirurgias de traumas panfaciais, em que há necessidade de manusear a oclusão do paciente no transoperatório, bem como em situações em que as técnicas consideradas mais convencionais, intubação orotraqueal e nasotraqueal, são contraindicadas. Além disso, atualmente pode ser indicada para cirurgia ortognática bimaxilar com rinoplastia simultânea, rinoplastia isolada e uma alternativa viável para pacientes portadores de fissura labial e/ou palatina. O objetivo deste trabalho é discutir as indicações e contraindicações deste método de intubação através de uma revisão de literatura, além de descrever os passos para sua execução.

Palavras-Chaves: Trauma Maxilofacial; Intubação; Submento-Oro-Traqueal ABSTRACT

The submental intubation or also known as submento-oro-traqueal is a technique that has conquered several maxillofacial surgeons over the years, in turn performed some small instances according to their anatomical knowledge and professional experience. It is a procedure considered safe, easy, fast and of low morbidity. For this reason it is used as an alternative to the widely used tracheostomy and has a higher chance of postoperative complications. Intubation is indicated for panacea trauma surgeries, in 
which there is a need to handle the occlusion of the patient in the intraoperative period, as well as in situations in which the techniques considered more conventional, orotracheal and nasotracheal intubation are contraindicated. In addition, it may currently be indicated for bimaxillary orthognathic surgery with simultaneous rhinoplasty, rhinoplasty alone and a viable alternative for patients with cleft lip and / or palate. The objective of this study is to discuss the indications and contraindications of this method of intubation through a literature review, in addition to describing the steps for its execution.

Key words: Maxillofacial Trauma; Intubation; Submento-Oro-Tracheal.

\section{INTRODUÇÃO}

Indivíduos que sofreram múltiplos traumas ósseos na face necessitam tratamento cirúrgico em ambiente hospitalar sob anestesia geral para redução das fraturas. Para esse procedimento ser realizado sob essas condições (YUSEON CHEONG, et al. 2016), se faz necessário manter o paciente ventilado durante a cirurgia. Existem diversas maneiras de manutenção das vias aéreas descritas na literatura que tornam possível a realização de cirurgias de fraturas panfaciais (ROCHA et al. 2006). As técnicas mais conhecidas são intubação oro-traqueal (IOT), intubação nasotraqueal (INT), traqueostomia (TQT) e intubação Submentoniana (ISM). 
Todas essas técnicas são perfeitamente aplicáveis em cirurgias bucomaxilofaciais, porém em determinadas situações, algumas podem ser contraindicadas. A IOT, por exemplo, não deve ser utilizada em situações em que é preciso analisar a relação mandíbula/maxila no transoperatório (YUSEON CHEONG, et al. 2016), pois o tubo ficaria localizado entre a face oclusal dos dentes. A INT não está indicada para fraturas cominutivas do terço médio da face (PETR SCHÜTZ, et al. 2008) porque o cirurgião teria dificuldades em diminuir a fratura. A TQT, único método indicado nos casos de fraturas faciais complexas até a criação da ISM, apresenta diversas complicações trans e pós - operatórias como hemorragias, estenose traqueal e comunicação traqueoesofágica (MANISALI M, et al. 2002; LESSARD MR, et al. 2000). Por essa razão sempre que possível, é ideal não utilizar essa técnica, dando preferência as com menor morbidade.

Quando há contraindicações para a IOT e a INT, que são as técnicas de intubação mais utilizadas, e se pretende evitar os possíveis problemas decorrentes da TQT, os profissionais podem lançar mão da ISM. Com essa técnica há a possibilidade de avaliar a relação oclusal durante a redução das fraturas como Le Fort I, II ou III, por exemplo, ou quando há fraturas na região nasal (RODRIQUEZ ED, et al. 1996; TOSTA M, et al. 2005; MAHMOOD S, LELLO GE 2002; ROCHA et al. 2016), visto que haveria dificuldades na passagem do tubo em uma INT . Além disso, atualmente a ISM está sendo usada para cirurgias não relacionadas com traumas como rinoplastia ou ortognática.

A intubação submento-oro-traqueal, também denominada de submentoniana, foi descrita pela primeira vez em 1986, por Francisco Hernandez Altemir, cirurgião BucoMaxilo espanhol (HERNANDEZ et al. 1986). Foi idealizada para substituir 
principalmente a TQT em virtude se sua alta morbidade (HERNANDEZ et al. 1986; PETR SCHÜTZ, et al. 2008; YUSEON CHEONG, et al. 2016). É considerada um procedimento seguro, fácil, rápido de baixa morbidade e proporciona boa visão do campo operatório (BARONE JR et al.2006; HAN JU, et al. 2003; MAHMOOD S, LELLO GE. 2002; ALIBERTI F, et al.2006).

\section{REVISÃO DE LITERATURA E DESCRIÇÃO DA TÉCNICA}

O método inicia-se após a intubação oro-traqueal (IOT) convencional, em que uma rigorosa antissepsia intra e extra-oral com P.V.P.I (Iodopovidona) 10\% ou mesmo com a utilização de clorexidina 2 ou 4\% é realizada, seguida de aposição dos campos cirúrgicos. Uma incisão na região sub-mentoniana de aproximadamente dois cm é realizada sob a pele (Figura A), onde após este plano o cirurgião deverá realizar uma divulsão através dos músculos platisma e milo-hióideo em direção à mucosa oral com a utilização de uma pinça hemostática curva do tipo Kelly ou tesoura de ponta romba do tipo metzenbaum (Figuras B e C). É muito importante que o profissional verifique se o acesso é suficiente para que o tubo a ser utilizado consiga entrar e ser manipulado com folga.

Sendo assim, após desconectar o tubo do aparelho, é através deste túnel que será realizada a passagem do cuff (Figura D) e posteriormente a do tubo aramado (Figura E) que se encontram na cavidade oral. Logo em seguida, o cirurgião deverá acoplar novamente o tubo ao aparelho de anestesia o mais rápido possível (Figura F).

Esta técnica é realizada com a utilização de um tubo aramado, a fim de evitar uma possível complicação, como a dobra do tubo, o que poderia implicar na pressão 
traqueal transoperatória devido a esta obliteração. Segundo PURICELLI 2003, o tubo endotraqueal aramado e siliconizado é o mais indicado, por oferecer grande maleabilidade sem comprometer a luz do tubo devido a dobras, trazendo riscos a este procedimento. LIM et al. 2003 recomendam a utilização de uma capa protetora na extremidade do tubo com o objetivo de evitar a introdução de sangue e outras secreções na luz do tubo durante a sua passagem pelo soalho da boca.

HERNANDEZ et al. 1986 propõe que o procedimento seja feito de modo que o tubo possa se mover de um lado a outro da língua, para que as manobras a serem realizadas no espaço intra-oral sejam facilitadas. Alguns cirurgiões fazem pequenas modificações na técnica original de Hernandez, baseados em suas vivências e preferências. ROCHA et al. 2006, por exemplo, aconselha que seja utilizado tubos de 7.0 ou 7.5 para intubação, por diminuir o risco de dobra.

Outro ponto na técnica que pode variar de um cirurgião para o outro é o local da incisão. Ainda de acordo com ROCHA et al. 2006, alguns autores orientam que a incisão seja feita lateral na região de corpo de mandíbula, mas que sua conduta é de fazer na região mediana. Essa escolha é feita por essa região não apresentar estruturas consideradas nobres e pela questão estética, visto que a cicatriz é menos visível.

Logo após o cuff ser inflado e o sistema ser restabelecido, o tubo pode ser fixado na região mentoniana com o auxílio de sutura com pontos simples, utilizando fio de nylon 3-0 ou 4-0 (CHIRADIP KAR et al. 2010). Esse procedimento faz com que o tubo permaneça estável diminuindo, dessa forma, riscos de extubação acidental, por exemplo.

\section{DISCUSSÃO}


De acordo com YUSEON CHEONG, et al. 2016 as pessoas que sofrem com múltiplos traumas ósseos na face precisam ser atendidos em ambiente hospitalar e de anestesia geral para que as fraturas sejam diminuídas. Desse modo, é preciso que seja mantida a ventilação dos pacientes. Com relação aos métodos de intubação, ROCHA et al. 2006 afirma que existem algumas técnicas conhecidas, possíveis de se usar nessas situações e que as mais utilizadas são a IOT e a INT. Informa ainda que em determinadas situações esses procedimentos são contraindicadas.

Com relação à IOT, ROCHA et al. 2006, CHIRADIP KAR et al. 2010 e PETR SCHÜTZ et al. 2008 entram em consenso sobre as contraindicações. Para eles esse procedimento não é aconselhável quando é preciso haver bloqueio maxilomandibular ou controlar a oclusão dentária no transoperatório. Isso ocorre porque o tubo utilizado para a passagem do oxigênio se posiciona entre a oclusal dos dentes. YUSEON CHEONG, et al. 2016 acrescenta que esse tipo de intubação dificulta a cirurgia, pois o acesso ao campo cirúrgico é diminuído em virtude da posição da cânula.

A respeito da INT, ROCHA et al. 2006 e CHIRADIP KAR et al. 2010 afirmam que essa técnica está contraindicada em casos de fraturas cominutivas do terço médio de face, porque podem causar obstrução física para a passagem da cânula pela narina, ou porque essa mesma cânula pode interferir negativamente na reconstrução de fraturas complexas naso-orbito-etmoidais. PETR SCHÜTZ et al. 2008 concorda e salienta que a INT não deve ser utilizada em lesões de face associadas com fratura de base de crânio, pois pode haver comunicação entre a cavidade nasal e a fossa craniana anterior, embora seja um fato raro. Outros pontos importantes são relacionados aos pólipos nasais, desvio de septo e patologias intranasais, que podem ser também consideradas contraindicações. 
Além disso, ainda sobre fraturas de base de crânio, YUSEON CHEONG et al. 2016 relata que a INT pode causar vazamento de fluído cerebrospinal e meningite.

ROCHA et al. 2006 reconhece que nos casos contraindicados para IOT e INT, existe outra alternativa para manutenção das vias aéreas, a traqueostomia (TQT). A TQT é considerada uma boa opção para pacientes com fratura panfaciais e aqueles que necessitem de maior tempo de ventilação. No entanto, reconhece também que essa técnica apresenta alta morbidade e com muitos riscos trans e pós-operatórios. Quanto aos riscos, MANISALI M, et al. 2002 e LESSARD MR, et al. 2000 indicam que estes podem ser hemorragias, estenose traqueal e comunicação traqueoesofágica. PETR SCHÜTZ at.al 2008 colabora expondo outros riscos como: danos a estruturas adjacentes, enfisema cervical, pneumotórax, pneumomediastino, bloqueio ou deslocamento da cânula, traqueíte, celulite, atelectasia pulmonar, fístula traqueal, traqueostomalácia e problemas estéticos com cicatrizes .

Pesquisas relatam que a TQT apresenta entre 14 e $45 \%$ de chances de resultar em algum tipo de complicação (SCHUTZ e HAMED 2008). Por conta da alta morbidade resultante dessa técnica, é preferível lançar mão de outra mais segura, quando possível. Além de tudo, CHIRADIP KAR et al. 2010 diz que a TQT é um procedimento que requer cuidados médicos mais meticulosos e maior tempo de permanência do paciente em ambiente hospitalar. Uma boa alternativa para a TQT, então, é a intubação submentoniana (ISM).

A ISM foi idealizada e descrita pela primeira vez por Hernández Altemir em 1986. A ideia era criar um método de intubação que pudesse substituir as IOT e INT, em casos contraindicados para as mesmas, e a TQT (HERNANDEZ et al. 1986; PETR SCHÜTZ, et al. 2008; YUSEON CHEONG, et al. 2016). A ISM veio ao longo dos anos 
ganhando aceitação em meio aos profissionais, que de acordo com suas experiências modificaram a técnica a fim de conseguir melhores resultados e até mesmo diminuir ainda mais sua morbidade.

Segundo ROCHA et al. 2006 e CHIRADIP KAR et al. 2010 após anos de pesquisa, estudos classificam a ISM como uma técnica simples, rápida e segura. Tais fatos são comprovados pelo baixo índice de complicações (no trans e/ou pósoperatórios) e de acidentes. De acordo com esses mesmos autores, a ISM é uma maneira de conciliar os benefícios da IOT e da INT, visto que permite a manipulação da oclusão dentária durante o procedimento cirúrgico, bem como acessar o terço médio da face em casos de fraturas frontonasais, por exemplo. Além de evitar problemas como meningite iatrogênica e suas complicações, que podem ocorrer, por exemplo, na técnica de INT e TQT. Outros benefícios da ISM evidenciados por YUSEON CHEONG, et al. 2016 são que o campo operatório fica mais visível, por causa da posição do tubo e que, se comparada a TQT, é naturalmente menos invasiva e com melhor estética, uma vez que a cicatriz se localiza em uma região menos aparente.

Apesar de ser usada largamente em casos de traumas, MACINNIS et al.1999 e CAUBI et al. 2008 argumentam que a ISM tem outras indicações como, por exemplo, cirurgia ortognática bimaxilar com rinoplastia simultânea, rinoplastia isolada, pacientes portadores de betatalasemia que necessitem de cirurgias ortognática. YUSEON CHEONG, et al. 2016 confirma essas informações e inclui outras indicações como casos de cancrum oris e também a utilização em alguns procedimentos realizados em base de crânio. Igualmente se mostra uma alternativa viável para o uso em pacientes portadores de lábio fissurado e/ou com fenda palatina. 
Ainda que considerada um método fácil, rápido e com baixa morbidade, a ISM pode apresentar algumas complicações e algumas contra indicações. A respeito das complicações, ROCHA et al. 2006 reitera que pode acontecer a dobra do tubo, podendo acarretar um aumento da pressão traqueal transoperatória. Algo possível de ser evitado quando se utiliza o tubo número 7 ou 7,5. Ainda relacionado às complicações, YUSEON CHEONG, et al. 2016 corrobora com esses dados anteriormente citados e adiciona que a saída acidental do tubo ( extubação) é a complicação mais severa já relatada, o que pode ser resguardado com uma firme sutura, procedimento já descrito por Hernandez na técnica original e também comumente utilizado na INT. Outros problemas como infecções cutâneas superficiais, parestesia temporária do nervo lingual, sangramento, cicatriz na região submentoniana (YUSEON CHEONG, et al. 2016), formação de abscesso no soalho de boca e sangramento (PETR SCHÜTZ, et al. 2008) eventualmente podem acontecer.

No tocante às contraindicações, ROCHA et al. 2006 e YUSEON CHEONG, et al. 2016 concordam que ISM não deve ser usada em pacientes que necessitem de tempo de ventilação prolongado (acima de 7 dias), que sejam expostos a repetidas cirurgias e indivíduos que tenham grave déficit neurológico e/ou traumas torácicos.

\section{CONCLUSÃO}

A intubação submentoniana é uma opção segura e de baixa morbidade, estando indicada em casos de fraturas múltiplas de face e/ou em situações em que a intubações convencionais são contraindicadas. Por depender apenas de materiais já disponíveis no armamentário cirúrgico padrão, se torna uma opção viável por sua realização não aumenta os custos do procedimento. É também uma técnica de rápida execução que, 
aliada ao conhecimento anatômico daquela região e a experiência do cirurgião que se proponha a executá-la, não afeta significativamente o tempo total em centro cirúrgico.

\section{Bibliografia}

1 - CHEONG Y, KIM J, et al. Submental Intubation for Complex Maxillofacial Injuries. Journal of Lifestyle Medicine. Vol. 6, No. 2, 68-71, 2016.

2 - ROCHA N, VASCONSELOS B, et al. Intubação submentoniana para manejo das vias aéreas em pacientes politraumatizado de face. Rev. Cir. Traumatol. Buco-MaxiloFac., Camaragibe v.6, n.3, p. 47 - 52, 2006.

3 - SCHÜTZ P, HAMED H. Submental Intubation Versus Tracheostomy. J Oral Maxillofac Surg 66:1404-1409, 2008.

4 - AMIN M, DILL-RUSSELL P, MANISALI M, et al. Facial fractures and submental tracheal intubation. Anaesthesia, 57(12):1195-9, 2002.

5 - CARON G, PAQUIN R, LESSARD MR, et al. Submental endotracheal intubation: an alternative to tracheotomy in patients with midfacial and panfacial fractures. $\mathrm{J}$ Trauma, 48: 235-40, 2000. 
6 - ADAMO AK, KATSNELSON T, RODRIQUEZ ED, et al. Intraoperative airway management with pan-facial fractures: alternative approaches. J Craniomaxillofac Trauma, 2(3):30-5, 1996.

7 - BRINHOLE MCP, TEIXEIRA R, TOSTA M, et al. Intubação submental: evitando a traqueostomia em cirurgia bucomaxilofacial. Rev Inst Ciênc Saúde, 23(2):169-72, 2005.

8 - MAHMOOD S, LELLO GE. Oral endotracheal intubation: median submental (retrogenial) approach. J Oral Maxillofac Surg. 60(4): 473-4, 2002.

9 - HERNANDEZ AF. The submental route for endotracheal intubation: a new tecnique. J Max Fac Surg, 14(1):64-5, 1986.

10 - JESUS AM, BARONE J, BARONE JR., Intubação oro-traqueal submentoneana: uma alternativa para fraturas múltiplas de face. Rev Méd IAMSPE. 31(1): 57 - 60, 2006.

11 - LIM HK, KIM IK, HAN JU, et al. Modified submental orotracheal intubation using the blue cap on the end of the thoracic catheter. Uonsei Med J. 44(5): 919-22, Oct. 2003.

12- TAGLIALATELA SC, MAIO G, ALIBERTI F, et al. Submento-submandibular intubation: Is the subperiosteal passage essential? Experience in 107 consecutives cases. J Oral Maxillofac Surg. 44(1):12-4, 2006.

13 - PURICELLI E. Intubação oro-traqueal corn urn trajeto submandibular: uma alternativa na traqueostomia eletiva. Rev Bras Cir Perio. 1(3): 238-41, 2003.

14 - KAR, CHIRADIP, MUKHERJEE, SRIJON. Submental Intubation: An Alternative and Cost-Effective Technique for Complex Maxillofacial Surgeries. J. Maxillofac. Oral Surg. (July-Sept 2010) 9(3):266-269, 2010.

15 - SCHÜTZ P; HAMED H. Submental Intubation Versus Tracheostomy in Maxillofacial Trauma Patients. J Oral Maxillofac Surg 66:1404-1409, 2008.

16 - MACINNIS, E.; BAIG, M. A modified submental approach for oral endotraeheal intubation. Int. J. oral Maxillofac. Surg., Copenhagen, v. 28, n.5, p. 344-6, oct., 1999.

17 - CAUBI AF, VASCONCELOS BCE, VASCONCELLOS RJH, MORAIS HHA, ROCHA NS. Submental intubation in oral maxillofacial surgery: Review of the literature and analysis of 13 cases. Med Oral Patol Oral Cir Bucal. 2008.

\section{Imagens}



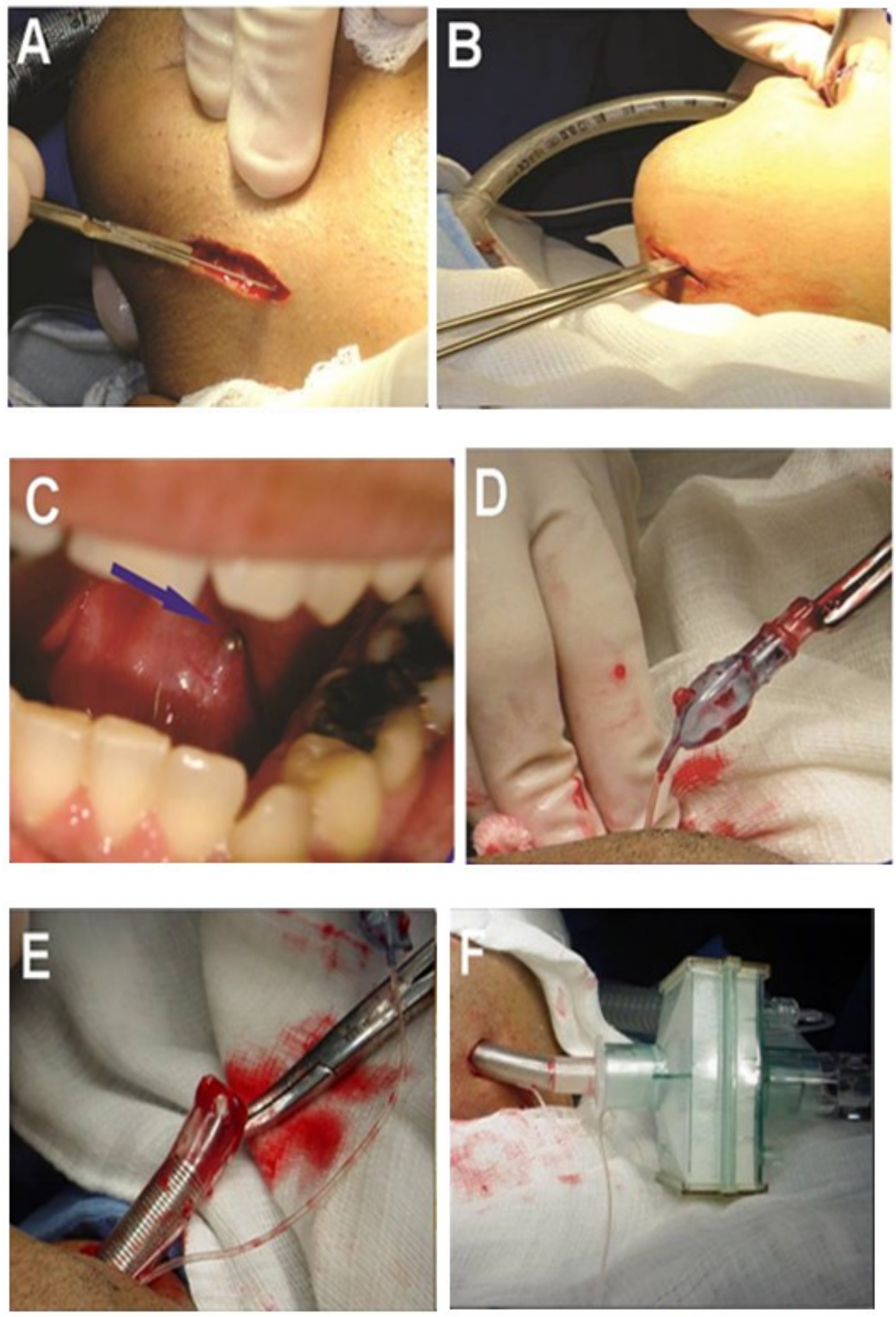

Figura A: Incisão de 2 cm em região submentoniana.

Figura B: Divulsão através dos planos anatôimicos.

Figura C: Visualização da ponta ativa da pinça Kelly em soalho bucal. 
Figura D: Passagem do cuff.

Figura E: Exteriorização do tubo.

Figura F: Acoplagem do tubo ao aparelho de anesthesia. 